\title{
Effects of maternal aging on localizations and expression levels of DNA methyltransferases and chromosome architecture in in-vivo matured mouse oocytes
}

\author{
TIAN Ning ${ }^{1}$, ZHANG Lu ${ }^{2}$, LÜ DanYu ${ }^{2}$, ZHENG JingGao ${ }^{1}$, MA WanYun ${ }^{1 *} \&$ LI Ying ${ }^{2}$ \\ ${ }^{1}$ State Key Laboratory of Low-Dimensional Quantum Physics, Department of Physics, Tsinghua University, Beijing 100084, China; \\ ${ }^{2}$ Department of Histology and Embryology, School of Basic Medical Sciences, Peking University Health Science Center, Beijing 100191, China
}

Received July 21, 2012; accepted October 30, 2012; published online July 12, 2013

\begin{abstract}
This paper investigated the age-related changes in the expression patterns of maintenance methyltransferase (DNMT1) and de novo methyltransferases (DNMT3a, 3b, 3L) and the chromosome architecture in in-vivo matured mouse oocytes using two-photon laser-scanning microscope. Our results showed that (1) DNMT1 and DNMT3a, 3b, 3L in the oocytes of pubertal mice were located in the cortical region of oocyte cytoplasm. In aging groups, DNMT1 was also located in the cortical region. However, DNMT3a, 3b, 3L had a relatively wider distribution in the oocyte cytoplasm and appeared near the chromosomes. These differences between pubertal and aging groups suggested that aging might affect DNA methylation; (2) the expression of DNMT1, and DNMT3a, 3b in aging groups increased significantly compared to pubertal groups, while, the expression of DNMT3L decreased. These results might be explained by the compensation mechanism among DNMTs, which might be impervious to aging; (3) aging caused increased errors in the distribution and three-dimensional morphology of chromosomes, including the increased total volume and surface area, the high ratio of height to diameter of a circular cylinder enclosing the chromosomes $(H / D)$. Our work provided morphological information for the studies of age-related decline in oocyte qualities.
\end{abstract}

aging, DNA methyltransferase, chromosome, oocyte, in vivo

Citation: Tian N, Zhang L, Lü D Y, et al. Effects of maternal aging on localizations and expression levels of DNA methyltransferases and chromosome architecture in in-vivo matured mouse oocytes . Chin Sci Bull, 2013, 58: 3248-3255, doi: 10.1007/s11434-013-5935-7

The number of elderly pregnancies is increasing. Generally, women experience a decline in their fertility around the age 30, and this decline is accelerated after the age 35. After reaching the age about 40, most women become infertile. Much research has investigated the link between maternal aging and the decline in female fertility. It has been demonstrated that this decline is a result of reduced oocyte reserves and poor oocyte quality induced by maternal aging [1-3]. In addition, the age-related degeneration in oocyte quality is often accompanied by epigenetic anomalies, increased errors in chromosome distribution, and disordered meiosis [2,4-8]. Clinical statistical results show that spontaneous abortion rates and teratism increase with age, both of which are factors affecting female fertility [9]. Basically,

*Corresponding author (email: mawy@mail.tsinghua.edu.cn) the miscarriage in elder women is primarily caused by the increase in chromosomal abnormalities in their oocytes, which consist of numerical and structural abnormalities. The most common examples of numerical abnormalities is monosomy and trisomy arising from a missing or an extra chromosome, and the structural abnormalities mainly refer to deletions, duplications, translocations, inversions and rings of part of a chromosome. Nevertheless, the exact reason for the increase in chromosomal abnormalities induced by maternal aging, remain unclear. Some studies find that this increase may be relevant to the spindle dysfunction, specific genetic mutation, epigenetic modification errors, and loss or degradation of chromosome-related proteins (e.g. cohesin) $[2,6,10]$.

Investigations into the mechanism of oocyte degeneration in older animals have uncovered a plethora of age- 
related genes $[1,11,12]$. Their abnormal transcriptional activity and expression are regulated by epigenetic modification [2]. DNA methylation is one of the normal mechanisms of endogenous modification. Gene methylation disorders induce genomic instability and significantly alter gene expression [13-15]. In mammalian cells, DNA methylation mainly involves the addition of a methyl $\left(\mathrm{CH}_{3}\right)$ group to the carbon 5 position of $\mathrm{CpG}$ dinucleotide. This important biochemical process is carried out by DNA methyltransferases (DNMTs) which use S-adenosyl methionine as the methyl donor. DNMTs can be divided into two general categories: de novo methyltransferases and maintenance methyltransferases. It is believed that DNMT3, three family members of which (DNMT3a, DNMT3b and DNMT3L) are known, is a de novo methyltransferase capable of methylating hemimethylated and unmethylated CpG. DNMT1 is considered to be the maintenance enzyme, which chiefly methylates hemimethylated $\mathrm{CpG}$ to copy DNA methylation patterns to the daughter strands during DNA replication [16-18]. In addition, it has been reported that the transcription levels of DNMT1, DNMT3a, DNMT3b, and DNMT3L, and their associated proteins, significantly changed in oocytes from aging mice [13]. Histone acetylation is another kind of normal endogenous modification and has been confirmed to have an association with chromosome structure [19]. Studies on histone modification found that the histone deacetylation level in the oocytes of older animals also changed [20,21]. Such changes in epigenetic modification, particularly DNA methylation and histone acetylation, could contribute to the chromosome aberration and disruption of meiosis in the oocytes of aging animals, and possibly to the progressive decline of female fertility.

Previous reports concerning DNMTs during mouse oocyte maturation have been focused mainly on the levels of gene expression or transcription, using molecular biological methods. However, there is still a lack of information on age-related DNMT protein expression. This paper aimed to further document the age-related changes in terms of morphology, including DNMT protein expression and chromosome architecture at the second meiotic metaphase (metaphase II or MII) in mature mouse oocytes using fluorescence staining. Their fluorescent images were collected by two-photon laser-scanning microscope (TPLSM). It employs two-photon absorption, which is a nonlinear optical effect, and uses a near-infrared femtosecond laser as its excitation source, to minimize scattering in the tissue and to increase the depth of penetration. Other advantages of the technique over conventional fluorescence microscopy include reduced phototoxicity, more efficient light detection, and a smaller photobleached area [22,23]. Additionally, DNMTs have been visualized using quantum dot 585 (QD 585) in our studies, which provides many advantages over traditional organic dyes, including much reduced photobleaching, strong anti-fluorescence quenching, wide absorption spectra, and narrow emission spectra [24-26]. There- fore, by applying TPLSM to QD-fluorescence in biological materials, we can obtain complementary fluorescent images of QD 585-labeled DNMTs and Hoechst 33342-counterstained chromosomes.

\section{Materials and methods}

\subsection{Animals}

Female Kun-Ming mice (Chinese Academy of Medical Sciences, Beijing, China) were housed and bred under standard conditions $\left(22 \pm 2^{\circ} \mathrm{C}, 55 \% \pm 5 \%\right.$ R.H., 12-h light/ dark cycle) with food and water available ad libitum, according to the Chinese National Standard (GB14925-2001). All animal experiments were approved by the Institutional Animal Welfares and Ethics Committee of Peking University (No. IRBLA2009-020).

\subsection{Collection of metaphase II-arrested mouse oocytes}

Female Kun-Ming mice, aged 7-8 weeks (pubertal) and 40-47 weeks (aging), were sacrificed by cervical dislocation. Oocytes arrested at the second meiotic metaphase (MII-arrested oocytes) were established by first injecting the mice with $10 \mathrm{IU}$ pregnant mare serum gonadotropin (Bo'en Pharmaceutical Ltd., Chifeng, China). After 48 h, this was followed by the injection of 10 IU human chorionic gonadotropin (Livzon Pharmaceutical Group Inc., Zhuhai, China), and the oocytes were harvested from the oviducts $15 \mathrm{~h}$ later. Cumulus cells were isolated by brief incubation in $0.05 \%$ hyaluronidase (Sigma, Louis, MO, USA; Cat. No. $\mathrm{H} 3506)$ at $37^{\circ} \mathrm{C}$ for $5 \mathrm{~min}$. MII-arrested nude oocytes were immediately fixed in $4 \%$ paraformaldehyde in phosphatebuffered saline (PBS) for $30 \mathrm{~min}$ at room temperature.

\subsection{Immunofluorescence}

Fixed MII-arrested oocytes of pubertal and aging mice were first permeabilized with $0.5 \%$ Triton X-100 for 30 min. After washed for three times in PBS, they were blocked in $2 \%$ normal goat or rabbit serum blocking solution for $30 \mathrm{~min}$. They were then incubated overnight at $4^{\circ} \mathrm{C}$ in $10 \mu \mathrm{L}$ droplets of polyclonal antibody, either rabbit anti-DNMT1, anti-DNMT3a, or anti-DNMT3b, or goat anti-DNMT3L (diluted 1:50 in PBS; Santa Cruz Biotechnology, Inc., Santa Cruz, CA, USA; Cat. Nos. sc-20701, sc-20703, sc-20704, sc-10239, respectively). After washing, the samples were immersed in a biotinylated goat anti-rabbit or rabbit antigoat IgG (diluted 1:100 in PBS; Jackson ImmunoResearch Laboratories, Inc., West Grove, USA) for $30 \mathrm{~min}$. Finally, the oocytes were rinsed and reacted with Quantum dot 585streptavidin conjugate (diluted 1:50 in PBS; Invitrogen, Carlsbad, CA, USA; Cat. No. Q10111MP) for $1 \mathrm{~h}$. Nuclei were counterstained with $5 \mu \mathrm{g} / \mathrm{mL}$ Hoechst 33342 (Molecular Probes, Leiden, The Netherlands) for $15 \mathrm{~min}$. The pri- 
mary antibody was omitted as the negative control.

\subsection{Two-photon fluorescence imaging system}

QD 585-labeled DNMTs and Hoechst 33342-labeled chromosomes were simultaneously observed using a Bio-Rad MRC 1024 system (Bio-Rad, Hercules, CA, USA) coupled to a Nikon TE300 inverted microscope (Nikon, Tokyo, Japan) with 100× oil objective (Plan Apochromat DIC H, NA 1.4; Nikon, Tokyo, Japan) (Figure S1). The excitation source was a collimated Ti: sapphire laser beam (Tsunami, Spectra-Physics, Mountain View, CA, USA) which was continuously tunable in the wavelength range of 720-1080 $\mathrm{nm}$, with a repetition rate of $82 \mathrm{MHz}$, and a pulse width of $120 \mathrm{fs}$. The laser beam of wavelength $730 \mathrm{~nm}$ was reflected into the back aperture of the objective by a short-pass dichroic mirror TS1 (625ESP; Chroma Technology, Bellows Falls, USA) and focused onto the specimen to induce the fluorescence of QD 585 and Hoechst 33342 simultaneously (Figure S2). The average power of the laser at the specimen plane was about $20 \mathrm{~mW}$ (peak power $1.5 \mathrm{~kW}$ ) in all experiment groups. The emission signals of the samples were collected by the same objective, passed through the main dichroic mirror TS1 and separated into two detection channels by dichroic mirror VHS (510DCLP; Chroma Technology). The transverse resolution was about $0.25 \mu \mathrm{m}(\mathrm{NA}=$ 1.4). The signals were collected by the two photomultiplier tubes (PMT). For grey level images $(512 \times 512)$, the scanning speed was set at $1 \mathrm{frame} / \mathrm{s}$. All the groups experienced the same experimental conditions (the same excitation laser power and wavelength, detector gain, etc).

\subsection{Image pre-processing}

By using Amira 5.2 (Visage Imaging, Berlin, Germany), the raw three-dimensional data of the fluorescent images were processed with a $3 \times 3 \times 3$ median filter to remove noise and then deconvoluted to restore the images. Subsequently, these images were segmented in an interactive way. The segmented fluorescent images were used to build the corresponding surface models.

\subsection{Image analysis: Definitions of statistical parameters}

Before formally introducing these parameters, we need to indicate that (1) The software Amira 5.2 was used to measure fluorescence intensity of DNMT protein, the total volume and surface area of chromosomes at metaphase II (MII-chros); (2) a self-edited program was used to calculate H/D.

The fluorescence intensity of DNMT protein was defined as the average grey value of QD 585-labeled DNMT in the whole spatial region occupied by the oocyte, which was assumed to be a semi-quantitative estimate of the DNMT protein expression level (i.e. lower grey values correspond- ed to lower expression levels). Although this was an imprecise estimate compared with that obtained by molecular-biological methods, we were able to simultaneously obtain accurate localization and a rough indication of the expression level of DNMT in a single oocyte without seriously destroying its morphology.

The total volume of MII-chros was defined as the volume of cytoplasm occupied by all 20 sister chromatids in the three-dimensional image of an MII-arrested mouse oocyte.

The total surface area was defined as the whole area of contact between the 20 sister chromatids and the cytoplasm.

Normally, the MII-chros of mouse oocytes are completely aligned in ordered ranks along the disc-shaped equatorial plate. This plate can be modeled as a short circular cylinder with the same volume and surface area to MII-chros (i.e. a short circular cylinder is used to enclose the MII-chros, and its volume and surface area are the same to the MII-chros). The flatness of the cylinder was then evaluated by the ratio of cylinder height to its underside diameter $(H / D)$, i.e. the smaller the ratio, the flatter the cylinder.

\subsection{Statistical analyses}

Values were expressed as means \pm SEM (SEM stands for the standard error of the mean). Statistical analyses were performed using SPSS 16.0 (SPSS Inc., Chicago, IL, USA). One-way ANOVA was used to compare the fluorescence intensity, the total volume and surface area of MII-chros, and $H / D$ ratio, between the pubertal and aging mice. The difference in the rates of chromosomal arrangement in pubertal mouse oocytes relative to the aging ones was analyzed with $C h i$-square statistics. $P<0.05$ was considered statistically significant.

\section{Results}

\subsection{DNMT protein expressions of MII-arrested oocytes in pubertal and aging mice}

The maintenance methyltransferase DNMT1 and the de novo methyltransferases DNMT3a, 3b, 3L in the oocytes of pubertal mice were localized in the cortical region of the oocyte cytoplasm (Figure 1(a)-(d)). In aging groups, DNMT1 was also localized in the cortical region (Figure 1(e)). However, DNMT3a, 3b, 3L were more widely distributed through the ooplasm and appeared near the chromosomes (Figure 1(f) $\left.-(h),\left(f^{\prime}\right)-\left(h^{\prime}\right)\right)$.

The fluorescence intensities of DNMT1 and DNMT3a, $3 b$ in the pubertal group were much lower than those in the aging group, but the fluorescence intensity of DNMT3L was much higher than those in the aging group (Figure 2). In summary, the localizations and expression levels of DNMT1 and DNMT3a, 3b, 3L were significantly altered in MII-arrested oocytes of aging mice. 


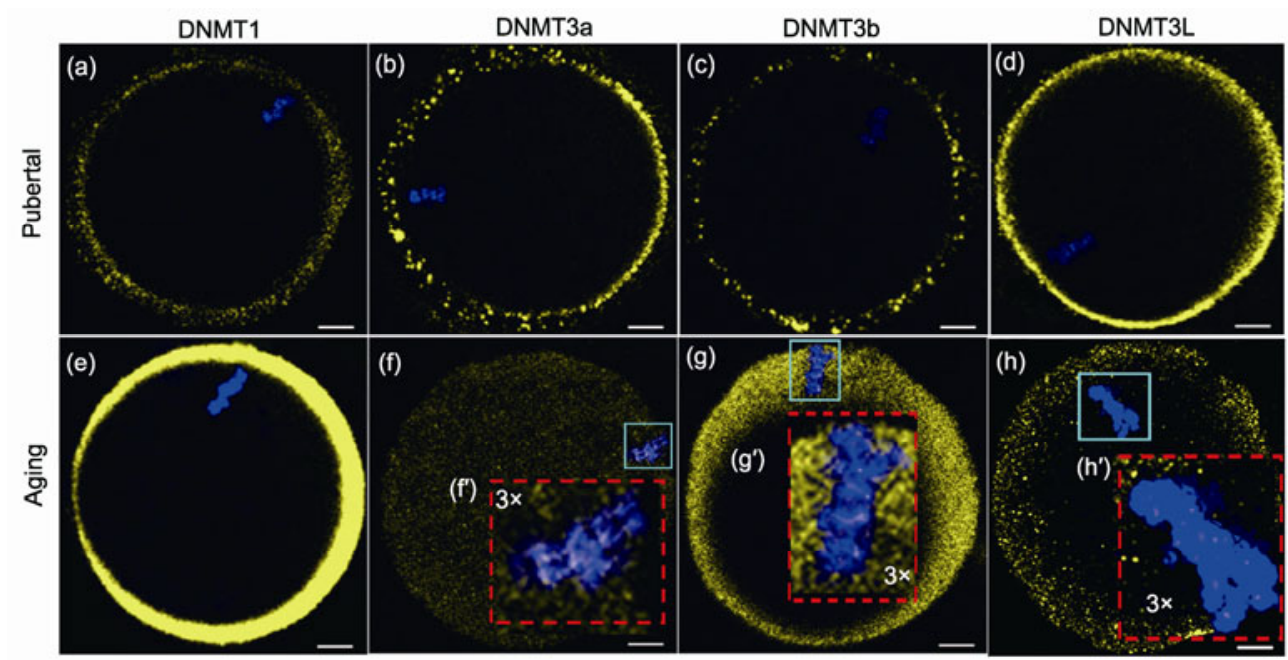

Figure 1 Immunofluorescent localizations of DNMTs (DNMT1 and DNMT3a, 3b, 3L) in MII-arrested oocytes of pubertal and aging mice. DNMTs and chromosomes of MII-arrested oocytes of pubertal ((a)-(d)) and aging ((e)-(h)) mice were labeled with QD 585 (yellow) and Hoechst 33342 (blue). Their typical micrographs (the maximally optically sectioned images) show the distribution of DNMT1 and DNMT3a, 3b, 3L. ( $\left.\mathrm{f}^{\prime}\right)$, ( $\left.\mathrm{g}^{\prime}\right)$, ( $\left.\mathrm{h}^{\prime}\right)$ show the localizations of DNMT proteins in the chromosomal region magnified three times. About 30 oocytes were observed in each group used. Scale bars $=15 \mu \mathrm{m}$.

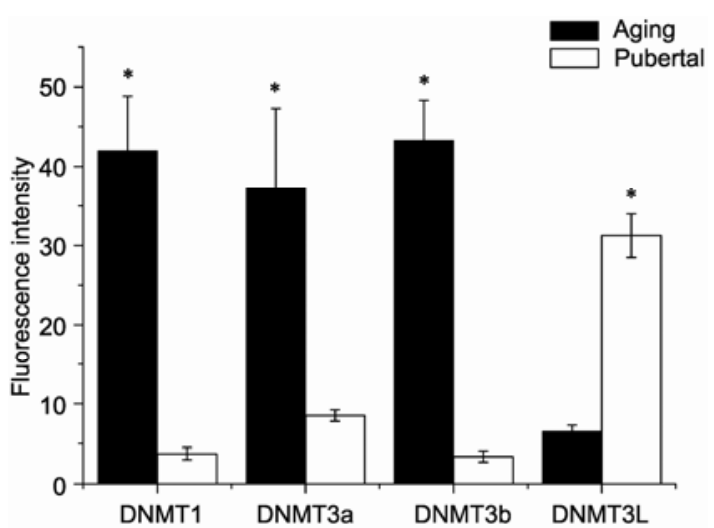

Figure 2 Fluorescence intensities of DNMT proteins in MII-arrested oocytes of pubertal and aging mice. The mark $(*)$ indicates a significant difference in the fluorescence intensity of DNMTs between pubertal and aging groups $(P<0.05)$. The standard error of the mean $(\mathrm{SEM})$ of each group is represented using error bar. About 30 oocytes were used in each group.

\subsection{Chromosome architecture of MII-arrested oocytes of pubertal and aging mice}

In the aging group, five chromosomal arrangements were observed: normal arrangement (Figure 3(A), (A')), nonrosette arrangement (Figure 3(B), $\left(\mathrm{B}^{\prime}\right)$ ), misaligned chromosomes (Figure 3(C), indicated by arrows), multiple metaphase plates (Figure 3(D), ( $\left.\mathrm{D}^{\prime}\right)$ ), and clumped arrangement (Figure $3(E),\left(E^{\prime}\right)$ ). In the pubertal group, three chromosomal arrangements, normal arrangement (Figure 3(a), 3( $\left.a^{\prime}\right)$ ), misaligned chromosomes (Figure 3(c), $\left(\mathrm{c}^{\prime}\right)$ ), and multiple metaphase plates (Figure 3(d), $\left(\mathrm{d}^{\prime}\right)$ ), were observed.

In the aging group, the normal arrangement of chromosomes is shown in Figure 3(A) and $\left(\mathrm{A}^{\prime}\right)$, which arrange themselves neatly along the equatorial plate Figure 3(A), and show a rosette-like distribution in the direction perpendicular to the metaphase plate Figure 3(A'). Several kinds of representative anomalies in chromosomal arrangements are listed in Figure 3(B)-(E), $\left(\mathrm{B}^{\prime}\right),\left(\mathrm{D}^{\prime}\right),\left(\mathrm{E}^{\prime}\right)$. (1) Non-rosette arrangement Figure $3(\mathrm{~B}),\left(\mathrm{B}^{\prime}\right)$ : chromosomes are characterized to arrange relatively regular as observed from the direction parallel to the metaphase plate Figure 3(B). However, observation in perpendicular direction shows an anomalous distribution Figure 3(B'); (2) misaligned chromosomes (Figure 3(C), indicated by the arrows): some chromosome is apart from the metaphase plate and keeps aloof, and the other chromosomes are still arranged in order; (3) multiple metaphase plates Figure 3(D), ( $\left.D^{\prime}\right)$ : chromosomes are irregular in formation, and they form into several ranks. Here, the example falls into two lines Figure 3(D). The triangles indicate the positions of the chromosomes out of alignment Figure 3(D); (4) clumped arrangement Figure 3(E), $\left(E^{\prime}\right)$ : all the chromosomes huddle together in a great mass, and it is hard to distinguish the metaphase plate. Thus, Figure 3(E) and $\left(E^{\prime}\right)$ are viewed from any two angles.

In the pubertal group, three kinds of chromosomal arrangements are observed: normal arrangement Figure 3(a), $\left(a^{\prime}\right)$, misaligned chromosomes (Figure 3(c), $\left(c^{\prime}\right)$, indicated by the arrows), and multiple metaphase plates Figure 3(d), $\left(d^{\prime}\right)$.

Therefore, there are more abnormal types of chromosomal arrangements in the aging group than in the pubertal group. The abnormal arrangements in the aging group may be more serious, e.g. the misaligned chromosomes of aging mice often wandered farther afield Figure 3(c).

The rate of normal arrangement was much lower in oocytes of aging mice (34\%) than in pubertal mice $(77 \%$, Table 1). Furthermore, the total volume and surface areas of the MII-chros, and the $H / D$ ratio were significantly greater in oocytes of aging mice than in the pubertal control group (Figure 4). Specifically, we estimated that the MII-chros in pubertal group had a total volume of $276.3 \mu^{3}$, a total 


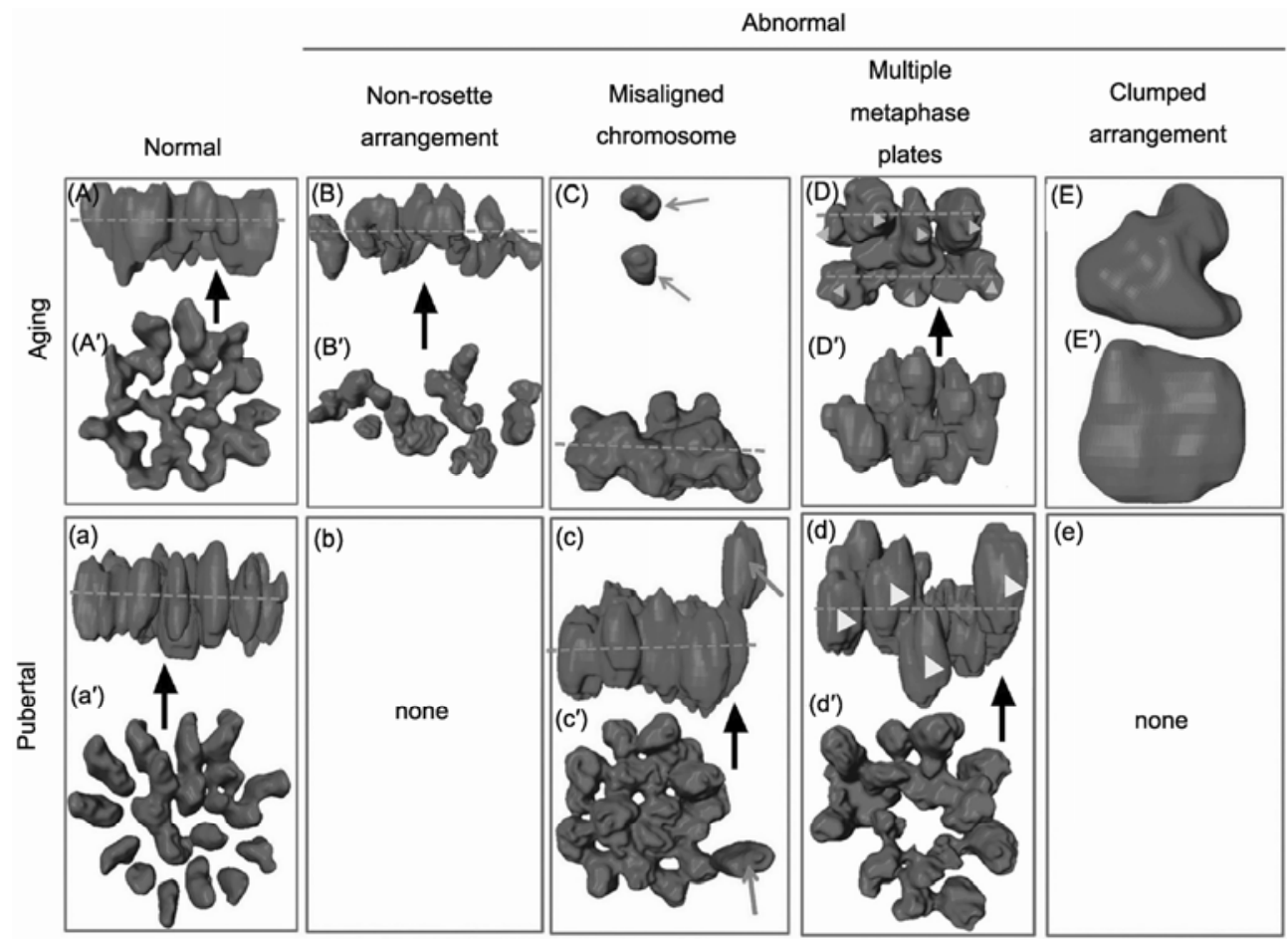

Figure 3 Chromosomal arrangements in MII-arrested oocytes of aging and pubertal mice. The chromosomes in MII-arrested oocytes were visualized using Hoechst 33342. They were reconstructed to restore their real three-dimensional forms using Amira 5.2, and their reconstructed images can be viewed from any angle. The observations from the direction parallel to the metaphase plate are shown in (A)-(D), (a)-(d), and the results in perpendicular direction (indicated by the black arrows $\uparrow$ ) are shown in $\left(\mathrm{A}^{\prime}\right),\left(\mathrm{B}^{\prime}\right),\left(\mathrm{D}^{\prime}\right),\left(\mathrm{a}^{\prime}\right),\left(\mathrm{c}^{\prime}\right),\left(\mathrm{d}^{\prime}\right)$. The image $(\mathrm{C})$ is only viewed from a parallel angle. The images $(\mathrm{E}),\left(\mathrm{E}^{\prime}\right)$ are viewed from any two angles. The dotted lines indicate the metaphase plate arranging the chromosomes.

Table 1 The rate of each chromosomal arrangement in the MII-arrested oocytes of the aging and pubertal mice ${ }^{\text {a) }}$

\begin{tabular}{ccc}
\hline Chromosomal arrangements & Aging $(\%)(n=33)$ & Pubertal $(\%)(n=65)$ \\
\hline Normal arrangement & $34(11 / 33)$ & $77(50 / 65)^{*}$ \\
Non-rosette arrangement & $21(7 / 33)$ & $0(0 / 65)$ \\
Misaligned chromosomes & $12(4 / 33)$ & $9(6 / 65)$ \\
Multiple metaphase plates & $27(9 / 33)$ & $14(9 / 65)$ \\
Clumped arrangement & $6(2 / 33)$ & $0(0 / 65)$ \\
\hline
\end{tabular}

a) $n$ is the number of the MII-arrested oocytes in each group. The percentages and numbers of oocytes with each chromosomal arrangement are shown. * The rate was significantly higher $(P<0.05)$ in the pubertal group than in the aging group.

surface area of $410.2 \mu \mathrm{m}^{2}$, and $H / D$ of 0.138 . Corresponding estimates for the aging group were $306.1 \mu \mathrm{m}^{3}, 477.6 \mu \mathrm{m}^{2}$, and 0.155 . The three parameters increased by $10.8 \%, 16.4 \%$, and $12.3 \%$, respectively.

\section{Discussion and conclusions}

\subsection{Age-related changes in the expression of mainte- nance methyltransferase, DNMT1}

In the oocytes of both aging and pubertal mice, the expression of DNMT1 protein was distributed in the cortical region. Aging may have limited effect on the localization of DNMT1. However, the fluorescence intensity of DNMT1 in the aging group was significantly higher than in the pubertal group, showing that its expression level was markedly elevated. It has been clearly shown that DNMT1 overexpression causes genomic hypermethylation, loss of imprinting, and embryonic lethality [27].

\subsection{Age-related changes in expressions of de novo me- thyltransferases, DNMT3a, DNMT3b and DNMT3L}

Both DNMT3a and DNMT3b in MII-arrested oocytes of aging mice were observed near the chromosomes. However, they were only localized in the cortical region in the pubertal group. Obviously, aging caused DNMT3a, $3 b$ in contact with chromosomes at metaphase II. It has been reported that DNMT proteins, which are synthesized and stored in the cytoplasm, only appeared in the nucleus when their enzymatic function was being carried out (i.e. when methylation was occurring), and subsequently moved back to the cytoplasm when they were no longer required [28]. In the aging group, the observation of DNMT3a, 3b near the chromosomes might imply that aging would cause aberrant methylation to occur.

It is extensively recognized that epigenetic reprogramming takes place during gametogenesis. In the development of mouse primordial germ cells, genome-wide demethylation first occurs, which erases the ancestry-imprinted genes. The genome is then remethylated according to the sex of the embryo and eventually resulting in the high methylation of 

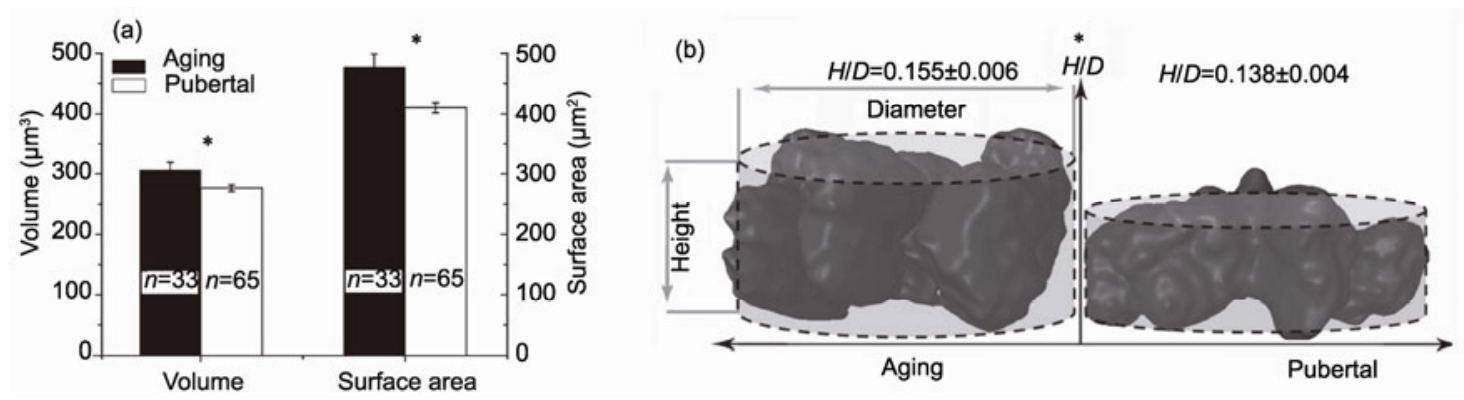

Figure 4 Three-dimensional morphology of the MII-chros in the oocytes of pubertal and aging mice. The total volumes and surface areas of the MII-chros are shown in (a); the ratio of height to diameter of a circular cylinder enclosing the MII-chros (H/D) is displayed in (b). All three parameters were significantly higher in the aging group than in the pubertal group $(*, P<0.05)$.

germ cells compared with the somatic cells [29,30]. The age-related disturbances in the localizations of DNMT3a, $3 \mathrm{~b}$ might affect the level of methylation in the mouse oocytes. It has been demonstrated that there is an age-related decrease of DNA methylation in different cow organs [31]. Therefore, the altered locations of DNMT3a, 3b could potentially cause aberrant methylation, disruption of genetic imprinting, aberrant expression, chromosomal instability, and other fatal effects [30,32,33].

Like DNMT3a and 3b, DNMT3L was located near the chromosomes in the aging group, and localized to the cortical region in the pubertal group. It is believed that DNMT3L is homologous to DNMT3a and DNMT3b but has no catalytic activity. It assists de novo methylation by increasing the ability of DNMT3a and $3 b$ to bind to DNA and to stimulate their activity [34]. The co-localization of DNMT3L and other DNMT3s might reflect this cooperative relationship of DNMT3 family. We also found that the fluorescence intensity of DNMT3L in MII-arrested oocytes of aging mice was obviously lower than in the pubertal group. This might be due to the age-related decline in the transcription level of DNMT3L [13]. Additionally, it was reported that the lack of DNMT3L, which participated in the establishment of the maternally imprinted genes, caused the degradation of oocyte quality, and finally brought about the death of early embryos [35]. To sum up, the reduced expression of DNMT3L protein, which was visualized semiquantitatively by its fluorescence intensity, might be another cause of the age-related decline in oocyte quality.

In addition, it was reported that the lack of either DNMT3L or DNMT1 in growing oocytes caused increased expression of de novo methyltransferase DNMT3b, suggesting a potential compensation mechanism in which the loss of one of the DNMTs would result in an increase of some others [35]. Our observations that in aging mice, the fluorescence intensities of DNMT1, DNMT3a, and DNMT $3 \mathrm{~b}$ were higher than in pubertal groups, while that of DNMT3L was much lower, were perhaps related to this compensation mechanism among the DNMT family. Alternatively, aging had limited effects on this mechanism.

\subsection{The effect of aging on chromosome architecture of MII-arrested mouse oocytes}

This paper indicated that aging strongly affected the morphology of MII-chros. Aged-related oocyte impairments are often accompanied by chromosome abnormalities and meiotic errors. It has been observed that, in the oocytes of elder women, one or more chromosomes were displaced from the metaphase plate during the second meiotic division [6]. It was also found that the rate of abnormal arrangement of chromosomes in the aging mouse oocytes was significantly increased [7,36]. These previous reports are quite consistent with the present mouse study. Our experimental results further demonstrated morphological changes, including increased total volume and surface area of MII-chros in the aging group. These changes might imply that, compared with the pubertal group, aging induced the chromatin strands to be less condensed, to occupy more space, and to present a larger area of contact with cytoplasm.

The eukaryotic chromatin is a complex of DNA, histones, non-histone proteins, and a small amount of RNA, tightly packed to form chromosomes. Epigenetic modifications of DNA and histones strongly affect the chromosome architecture. It has been reported that DNA methylation is essential for chromatin remodeling and for genomic imprinting and such changes may be associated with altered chromosome architecture [30,37,38]. Histone acetylation decreases their affinity to DNA and causes a change in nucleosome conformation and increased transcriptional activity [39], and histone deacetylation is critical for oocyte differentiation and chromosome segregation [38]. Moreover, the progression of histone deacetylation was decreased in oocytes of aging mice, resulting in some acetylation remaining, and eventually causing aneuploidy and embryo death [20]. Considering the important roles of DNA and histone modification, we speculate that the altered morphology, expanded volume and increased surface area of MII-chros, may be the macroscopic results of inadequate histone deacetylation, altered DNA methylation, or perhaps other factors.

This work provides age-related morphological changes in DNMT expression and chromosome architecture at meta- 
phase II of mouse oocytes. The altered DNMT expression may directly or indirectly affect the chromosome architecture through DNA methylation. Actually, the relationship between them still needs to be further studied.

\subsection{Conclusions}

It is indisputable that maternal age is responsible for abnormal changes in epigenetic modification and chromosome abnormality at birth. This work aimed to directly observe the macroscopic effects of aging on DNMT proteins catalyzing the DNA methylation and chromosome architectures using fluorescence staining. We have made two important observations. The first was that aging interfered with the localizations and expression levels of DNMT proteins and might affect DNA methylation in mouse oocytes. Second, the rate of age-related error in chromosomal arrangement was increased, as indicated by changed morphology, volume and surface expansion, and the less condensed form of MII-chros. These morphological changes in DNMT proteins and MII-chros might reflect interrelationships between them and, presumably, underlie the effects of maternal age on oocyte quality. This paper provides experimental justification for further morphological research on the age-related decline in female fertility and will assist better understanding of molecular biological information in this field.

This work was supported by the National Natural Science Foundation of China (90919012 and 10874099), National Basic Research Program of China (2007CB5119004), the Doctoral Program Research Fund of the Chinese Ministry of Education (20090002110065), and the Tsinghua University Initiative Scientific Research Program (2010THZ01).

1 Velde E R, Pearson P L. The variabiligy of female reproductiove aging. Hum Reprod Update, 2002, 8: 141-154

2 Lopes F L, Fortier A L, Darricarrère N, et al. Reproductive and epigenetic outcomes associated with aging mouse oocytes. Hum Mol Genet, 2009, 18: 2032-2044

3 Tatone C. Oocyte senescence: A firm link to age-related female subfertility. Gynecol Endocrinol, 2008, 24: 59-63

4 Burzynski S R. Gene silencing-A new theory of ageing. Med Hypotheses, 2003, 60: 578-583

5 De La Fuente R. Chromatin modifications in the germinal vesicle (GV) of mammalian oocytes. Dev Biol, 2006, 292: 1-12

6 Battaglia D E, Goodwin P, Klein N A, et al. Influence of maternal age on meiotic spindle assembly in oocytes from naturally cycling women. Hum Reprod, 1996, 11: 2217-2222

7 Liu L, Keefe D L. Ageing-associated aberration in meiosis of oocytes from senescence-accelerated mice. Hum Reprod, 2002, 17: 26782685

8 Eichenlaub-Ritter U, Staubach N, Trapphoff T. Chromosomal and cytoplasmic context determines predisposition to maternal agerelated aneuploidy: Brief overview and update on MCAK in mammalian. Biochem Soc Trans, 2010, 38: 1681-1686

9 Ottolenghi C, Uda M, Hamatani T, et al. Aging of oocyte, ovary, and human reproduction. Ann NY Acad Sci, 2004, 1034: 117-131

10 Lelanda S, Nagarajan P, Polyzos A, et al. Heterozygosity for a Bub1 mutation causes female-specific germ cell aneuploidy in mice. Proc Natl Acad Sci USA, 2009, 106: 12776-12781

11 Pan H, Ma P P, Zhu W T, et al. Age-associated increase in aneu- ploidy and changes in gene expression in mouse eggs. Dev Biol, 2008, 316: 397-407

12 Semsei I. On the nature of ageing. Mech Ageing Dev, 2000, 117: $93-$ 108

13 Hammatani T, Falco G, Carter M G, et al. Age-associated alteration of gene expression patterns in mouse oocytes. Hum Mol Genet, 2004, 13: $2263-2278$

14 Sato A, Otsu E, Negishi H, et al. Aberrant DNA methylation of imprinted loci in superovulated oocytes. Hum Reprod, 2007, 22: 26-35

15 Kulis M, Esteller M. DNA methylation and cancer. Adv Genet, 2010, 70: 27-56

16 Goll M G, Bestor T H. Eukaryotic cytosine methyltransferases. Annu Rev Biochem, 2005, 74: 481-514

17 Luczak M W, Jaqodziński P P. The role of DNA methylation in cancer development. Folia Histochem Cytobiol, 2006, 44: 143-154

18 Miyake T, Endo K, Honjo S, et al. Expression of DNA methyltransferase (DNMT) 1, 3a and 3b proteins in human hepatocellular carcinoma. Yonago Acta Med, 2010, 53: 1-7

19 Bannister A J, Kouzarides T. Regulation of chromatin by histone modifications. Cell Res, 2011, 21: 381-395

20 Akiyama T, Nagata M, Aoki F. Inadequate histone deacetylation during oocyte meiosis causes aneuploidy and embryo death in mice. Proc Natl Acad Sci USA, 2006, 103: 7339-7344

21 van den Berg I M, Eleveld C, van der Hoeven M, et al. Defective deacetylation of histone $4 \mathrm{~K} 12$ in human oocytes is associated with advanced maternal age and chromosome misalignment. Hum Reprod, 2011, 26: 1181-1190

22 So P T C, Dong C Y, Masters B R, et al. Two-photon excitation fluorescence microscopy. Annu Rev Biomed Eng, 2000, 2: 399-429

23 Xu C, Zipfel W, Shear J B, et al. Multiphoton fluorescence excitation: New spectral windows for biological nonlinear microscopy. Proc Natl Acad Sci USA, 1996, 93: 10763-10768

24 Michalet X, Pinaud F F, Bentolila L A, et al. Quantum dots for live cells, in vivo in aging and diagnostics. Science, 2005, 307: 538-544

25 Walling M A, Novak J A, Shepard J R E. Quantum dots for live cell and in vivo imaging. Int J Mol Sci, 2009, 10: 441-491

26 Drummen G P. Quantum dots-from synthesis to applications in biomedicine and life sciences. Int J Mol Sci, 2010, 11: 154-163

27 Biniszkiewicz D, Gribnau J, Ramsahoye B, et al. Dnmt1 overexpression causes genomic hypermethylation, loss of imprinting, and embryonic lethality. Mol Cell Biol, 2002, 22: 2124-2135

28 Lees-Murdock D J, Shovlin T C, Gardiner T, et al. DNA methyltransferase expression in the mouse germ line during periods of $d e$ novo methylation. Dev Dyn, 2005, 232: 992-1002

29 Reik W, Dean W, Walter J. Epigenetic reprogramming in mammalian development. Science, 2001, 293: 1089-1093

30 Bestor T H. The DNA methyltransferases of mammals. Hum Mol Genet, 2000, 9: 2395-2402

31 Zin' Kovskaia G G, Berdyshev G D, Vaniushin B F. Tissue-specific decrease and change in the character of DNA methylation in cattle with aging. Biokhimiia, 1978, 43: 1883-1892

32 Turek-Plewa J, Jagodzinski P P. The role of mammalian DNA methyltransferases in the regulation of gene expression. Cell Mol Biol Lett, 2005, 10: 631-647

33 Pradhan S, Esteve P O. Mammalian DNA (cytosine-5) methyltransferases and their expression. Clin Immunol, 2003, 109: 6-16

34 Suetake I, Shinozaki F, Miyagawa J, et al. DNMT 3L stimulates the DNA methylation activity of Dnmt 3a and Dnmt $3 \mathrm{~b}$ through a direct interaction. J Biol Chem, 2004, 279: 27816-27823

35 Lucifero D, Salle S L, Bourc'his D, et al. Coordinate regulation of DNA methyltransferase expression during oogenesis. BMC Dev Biol, 2007, 7: 36-50

36 Takeda T, Hosokawa M, Higuchi K. Senescence-accelerated mouse (SAM): A novel murine model of senescence. Exp Gerontol, 1997, 32: $105-109$

37 Margot J B, Ehrenhofer-Murray A E, Leonhardt H. Interactions within the mammalian DNA methyltransferase family. BMC Mol Biol, 2003, 4: 7-15

38 Matarazzo M R, Boyle S, D’Esposito M, et al. Chromosome territory 
reorganization in a human disease with altered DNA methylation. Proc Natl Acad Sci USA, 2007, 104: 16546-16551
39 Struhl K. Histone acetylation and transcriptional regulatory mechanisms. Genes Dev, 1998, 12: 599-606

Open Access This article is distributed under the terms of the Creative Commons Attribution License which permits any use, distribution, and reproduction in any medium, provided the original author(s) and source are credited.

\section{Supporting Information}

Figure S1 Experimental setup for two-photon fluorescence imaging.

Figure S2 Absorption and fluorescence emission spectra of QD 585 (a) and Hoechst 33342 (b).

The supporting information is available online at csb.scichina.com and www.springerlink.com. The supporting materials are published as submitted, without typesetting or editing. The responsibility for scientific accuracy and content remains entirely with the authors. 